\title{
A compendium of molecules involved in vector-pathogen interactions pertaining to malaria
}

\author{
Sreelakshmi K Sreenivasamurthy ${ }^{1 \dagger}$, Gourav Dey ${ }^{1 \dagger}$, Manjula Ramu', Manish Kumar ${ }^{1}$, Manoj K Gupta ${ }^{1}$, \\ Ajeet K Mohanty ${ }^{2}$, HC Harsha' ${ }^{1}$ Pushkar Sharma ${ }^{3}$, Nirbhay Kumar ${ }^{4}$, Akhilesh Pandey ${ }^{5}$, Ashwani Kumar ${ }^{2}$ \\ and TS Keshava Prasad ${ }^{1 *}$
}

\begin{abstract}
Malaria is a vector-borne disease causing extensive morbidity, debility and mortality. Development of resistance to drugs among parasites and to conventional insecticides among vector-mosquitoes necessitates innovative measures to combat this disease. Identification of molecules involved in the maintenance of complex developmental cycles of the parasites within the vector and the host can provide attractive targets to intervene in the disease transmission. In the last decade, several efforts have been made in identifying such molecules involved in mosquito-parasite interactions and, subsequently, validating their role in the development of parasites within the vector. In this study, a list of mosquito proteins, which facilitate or inhibit the development of malaria parasites in the midgut, haemolymph and salivary glands of mosquitoes, is compiled. A total of 94 molecules have been reported and validated for their role in the development of malaria parasites inside the vector. This compendium of molecules will serve as a centralized resource to biomedical researchers investigating vector-pathogen interactions and malaria transmission.
\end{abstract}

Keywords: Knockdown, RNAi, Gene silencing, Plasmodium, Anopheles, Oocyst, Sporozoite

\section{Background}

Malaria continues to be one of the most debilitating mosquito-borne diseases. According to the WHO World Malaria Report in 2011, 216 million cases of malaria were reported in the year 2010, resulting in 655,000 deaths, of which $86 \%$ were children below five years of age. A total of 41 different species of Anopheles mosquitoes act as vectors for transmission of human malaria [1]. Around 19 of them have been found to play a major role in disease-transmission across Asia-Pacific [2]. Increasing instances of pathogens gaining resistance to the first-line of drugs and replacement anti-malarials have complicated the scenario of disease control in endemic areas. One of the approaches adopted towards limiting the spread of disease is to control the vector population by the reduction of breeding sites and use of insecticides and by minimizing mosquito-human contacts through

\footnotetext{
* Correspondence: keshav@ibioinformatics.org

${ }^{\dagger}$ Equal contributors

'Institute of Bioinformatics, International Technology Park, Bangalore 560066, India

Full list of author information is available at the end of the article
}

the use of mosquito nets impregnated with insecticides. Though these measures have been largely effective, the development of resistance to insecticides coupled with the increased adaptability of the pathogen susceptible strains of vectors have emphasized the need for the development of alternative approaches to intervene the transmission of malaria [3].

In their complex life cycle, during sporogonic development malaria parasites are in intimate contact with midgut, haemolymph and salivary glands of vector mosquito. The parasite, on its entry through the blood meal has to traverse through the midgut of mosquito, which acts as the first physical barrier inside the vector. Invasion of the midgut epithelia by the ookinete evokes responses within the vector to counter the assault. This constitutes the initial checkpoint for successful establishment of pathogen within the vector. The ookinetes then form oocysts, which mature in the midgut basal lamina for the next 10-12 days. Development of sporozoites heavily relies upon the mosquito metabolism. Upon maturation, each oocyst ruptures to release thousands of sporozoites into the haemocoel (Figure 1). Here, the sporozoites encounter the

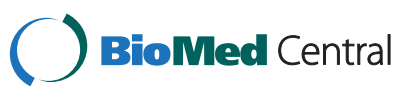




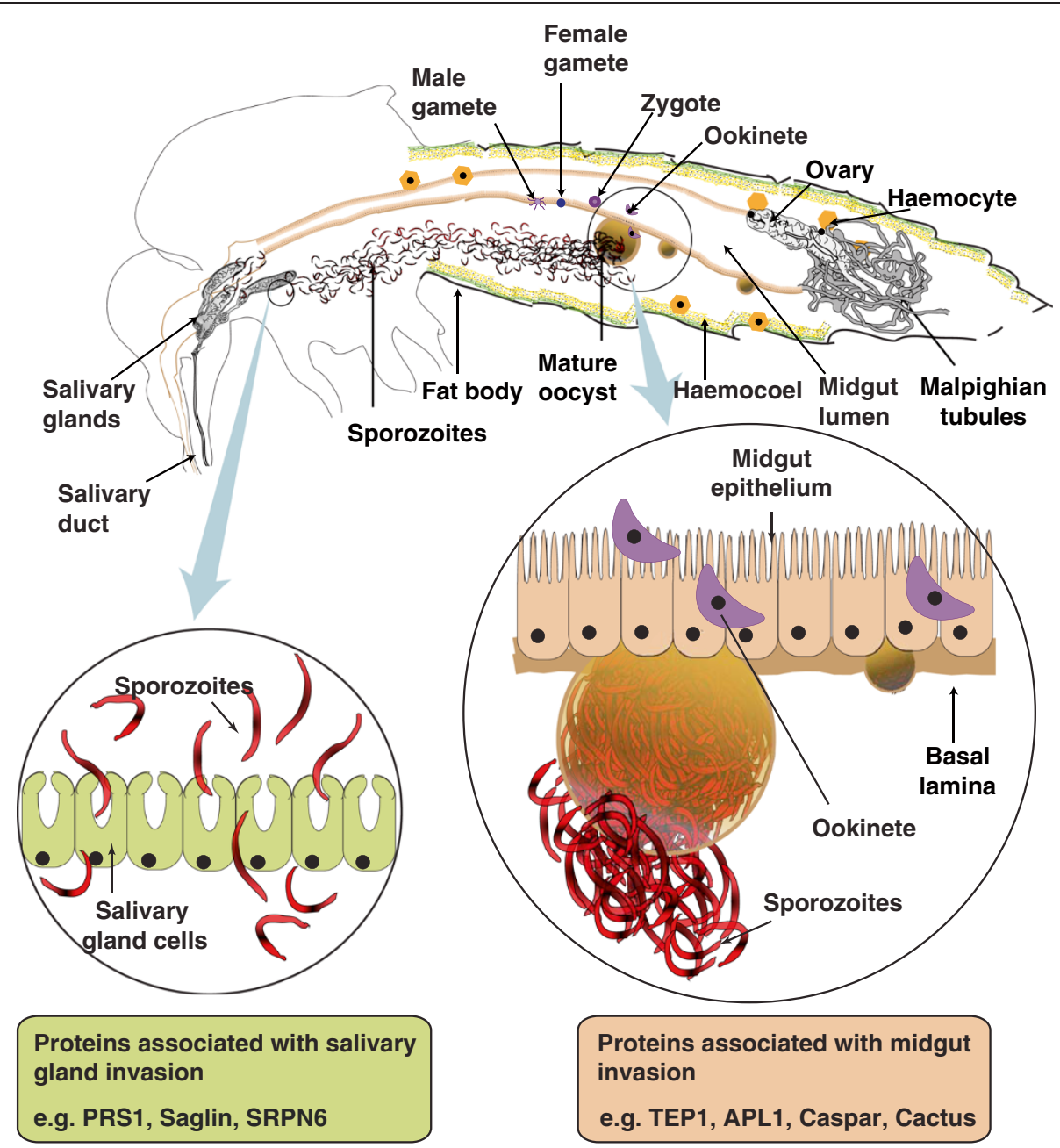

Figure 1 Plasmodium life-cycle within the mosquito. Ingestion of Plasmodium infected blood by the mosquitoes is followed by reductional division in male and female gametocytes resulting in formation and fusion of male and female gametes within mosquito midgut, to form a zygote. The zygote then develops into an ookinete. The ookinete invades midgut epithelia to reach basal lamina, where it matures into oocyst. The oocysts upon maturation, release hundreds of sporozoites into haemocoel. Sporozoites migrate from midgut to salivary gland through haemolymph and then invade salivary glands. Once inside the salivary gland the sporozoites undergo further modifications and are injected into the host blood stream during a blood meal.

second challenge by the host haemocytes. The surviving sporozoites then travel to the salivary glands and gain entry into the salivary glands to undergo further modifications that are necessary to infect the subsequent mammalian host.

It is known that specific proteins mediate the interactions of the Plasmodium parasites with mosquito tissues and regulate their maturation within their host vectors [4-6]. For example, epithelial serine protease (ESP) and cactus proteins in the midgut are known to promote the entry of Plasmodium ookinetes into the midgut lamina, while thioester-containing protein 1 (TEP1) and serine protease inhibitor 6 (SRPN6) proteins inhibit this process [7-12]. Similarly, the binding and invasion of salivary glands by Plasmodium sporozoites is reported to be mediated by the interaction of a set of surface proteins. Saglin, a receptor on salivary gland, which binds to thrombospondin-related anonymous protein on sporozoites, is one such protein involved in the internalization of sporozoites into the salivary glands of mosquito-vectors $[13,14]$. The difference in the vector competence of the Anopheles species may in part be determined by the expression of such proteins involved in the vector-pathogen interactions. RNA interference based gene silencing techniques have been successfully used in determining the role of several such mosquito proteins in the invasion of vector by malaria parasite. There has been a considerable increase in the use of this technique in the recent years (Figure 2) and many mosquito proteins, which have a role in growth and survival of malaria parasites inside the 


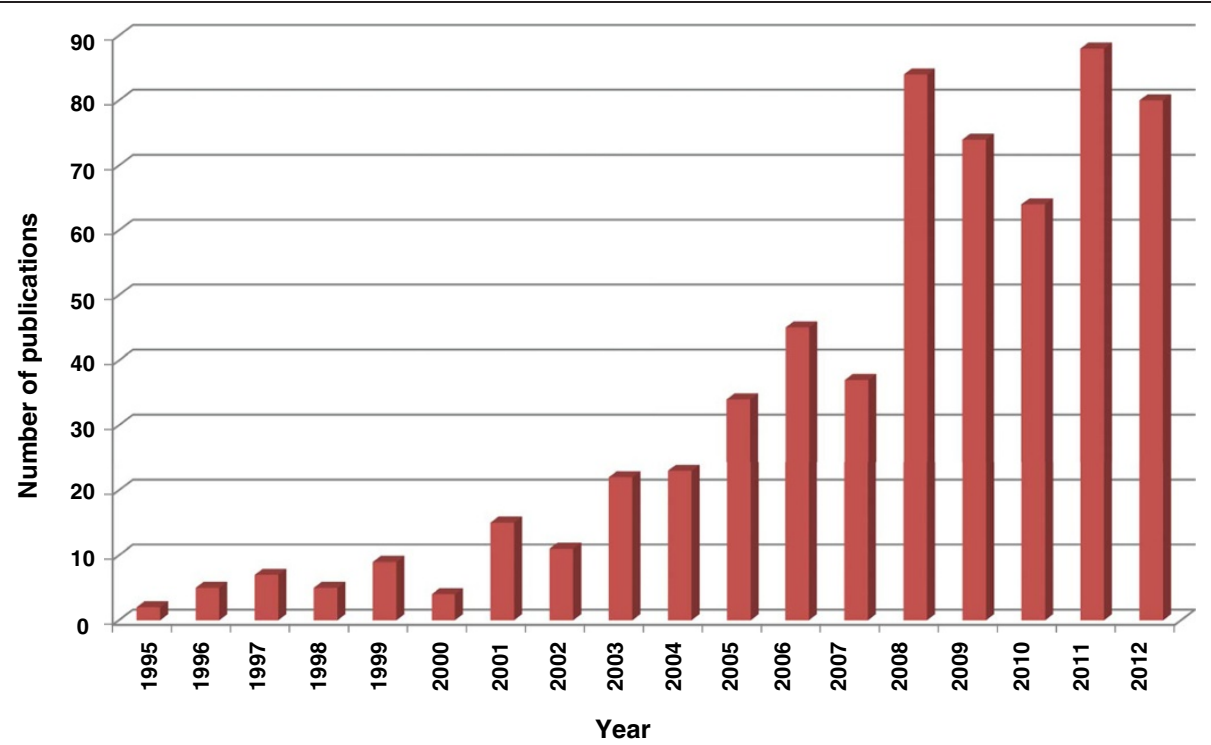

Figure 2 Trend of knockdown studies in mosquitoes. There has been an increasing trend towards knockdown studies carried out in mosquitoes recently. The graph shows a 10-fold increase in the use of gene silencing approaches to validate the role of proteins in vector-pathogen interactions.

vector, have been reported. Therefore, a literature survey was performed to gather and catalog mosquito proteins with an established role in parasite development. Such a resource will be helpful in future investigations on candidate targets for strategies aimed at preventing malaria transmission.

\section{Methods}

Scientific literature was searched for molecules involved in infection of the vector by Plasmodium species. In addition to a keyword search for knockdown studies in mosquitoes, several author specific searches were also performed to retrieve articles relevant to vector-pathogen interactions. These were then screened for articles reporting the effect of RNA silencing on the rate of infection. Articles with the reports of RT-PCR experiments, which had further verified the reduction of mRNA expression, were also considered for annotation. From these articles, proteins that resulted in significant changes in the rate of infection upon gene silencing were annotated. These proteins were further segregated into three major groups - i) molecules involved in midgut invasion; ii) molecules involved in melanization; and iii) molecules involved in salivary gland invasion. Molecular conditions brought about by gene silencing events, which resulted in increased numbers of oocysts at midgut lamina and sporozoites at salivary glands, were inferred to promote parasite growth and the corresponding gene or genes were deemed to be inhibitory to parasite growth. Similarly, increased melanization of ookinetes was referred to as negative effect on parasite growth.

\section{Summary of molecules involved in vector-Plasmodium interactions}

This article presents a resource of 94 evidence-based molecules involved in vector-pathogen interactions and vector immune responses. From an initial list of 572 articles retrieved using different search strategies, a subset of 55 articles contained reports of vector-pathogen interactions, from which the information was compiled into this compendium. Proteins, either promoting or inhibiting parasite growth were catalogued with associated information on the number of oocysts produced, rate of melanization of ookinetes in midgut and/or number of sporozoites found in the salivary glands upon different experimental conditions (Figure 3). The most common method employed to determine the role of mosquito protein infection was to knockdown the gene expression and then measure its effect in terms of oocyst count. Among 78 proteins identified by this strategy, oocyst count decreased on silencing 33 genes and increased in case of 45 genes (Figure 4). In other words, former set of proteins were found to promote midgut invasion whereas the latter to result in inhibition. Only six studies reported investigation of proteins, which are involved in salivary gland invasion (Figure 4). At least 22 molecules were found to affect the melanization process upon silencing. Knocking down of 13 and 9 genes resulted in increased and decreased melanization, respectively (Figure 4). Some of these molecules had no effect on either the oocyst or sporozoite counts, when silenced individually. However, they showed significant variation when silenced in combination with other genes. The role of certain proteins, including gastrin/cholecystokinin receptor 1 


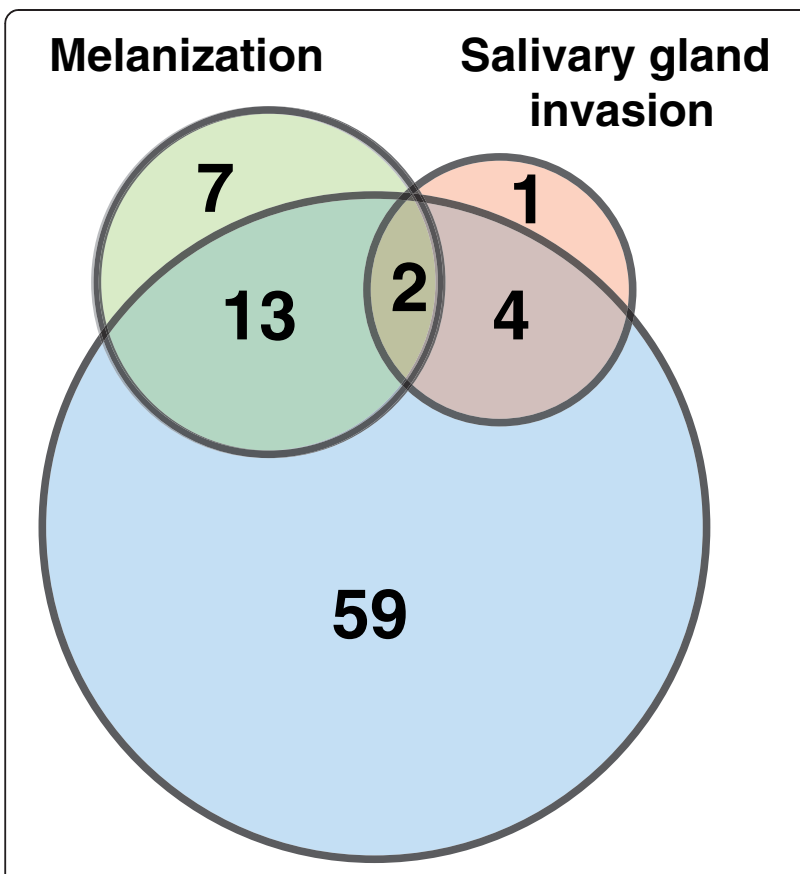

\section{Midgut invasion}

Figure 3 Venn diagram representation of molecules involved in mosquito-Plasmodium interactions at various stages. A total of 78 molecules were validated for their role in the midgut invasion, 22 in melanization and 7 in salivary gland invasion. Among these, SRPN6 and TEP1 were found to be involved in midgut and salivary gland invasion along with melanization of ookinetes. 13 proteins were found to be involved both in the midgut invasion and melanization and 4 molecules were involved in both midgut and salivary gland invasion.

(GPRCCK1) and leucine-rich repeat immune protein (LRIM1), was dependent on the species of mosquitoes in which the experiment was performed and the Plasmodium species used for infecting them [15-17].

\section{Molecules involved in midgut invasion}

Fusion of male and female gametes within the midgut of the mosquitoes results in the formation of zygotes and ookinetes, which then invade epithelium of the midgut and migrate to the basal lamina to form oocysts. The invasion of mosquito midgut by pathogens provides a critical step that involves adhesion to the midgut epithelia of the vector. Successful recognition and invasion of midgut epithelia by ookinetes is dependent on molecules that either facilitate or inhibit the parasite development. For instance, alanyl aminopeptidase N (APN1) was reported as a surface recognition molecule in the mosquito midgut [18]. Epithelial invasion by the ookinetes is followed by the transformation of ookinetes to oocysts in the basal lamina. Seventy eight mosquito proteins were shown to be involved in midgut invasion by ookinetes and parasite survival, by survey of scientific literature.

Proteins such as TEP1 and LRIM1 are known to recognize the invading ookinetes and trigger immune responses in mosquitoes (Additional file 1) $[8,16,19,20]$. Ctype lectin 4 (CTL4), caspar and cactus are found to negatively regulate the immune responses, resulting in a decreased oocyst count upon their silencing [8,21]. The proteins which generated complete refractoriness in the vector upon silencing may be the target of choice for future studies to block disease transmission. Heat shock protein, HSC3 resulted in decreased oocyst count upon knocking down in case of Anopheles gambiae and Plasmodium falciparum. However, in case of Anopheles stephensi infected with Plasmodium yoelii, it resulted in increased oocyst count [16]. Similarly gastrin/cholecystokinin receptor 1 (GPRCCK1) also showed increase in the oocyst count upon infection of An. gambiae with Plasmodium berghei and decrease upon infection with $P$. falciparum (Additional file 2) [15].

Certain proteins such as clip-domain serine protease 17 (CLIPB17) showed no significant change in the number of oocysts upon its silencing. However, in combination with CTL4, silencing of CLIPB17 resulted in a significant decrease of oocysts (Additional files 1 and 3) [21]. Similarly, ARC P21 and ARC P41 subunits of the actin related protein complex shows no effect on the number of oocysts formed, when silenced individually. However, a double knockdown of these genes resulted in the formation of larger number of oocysts [22].

\section{Molecules involved in melanization}

Of the many ookinetes that are formed in the mosquito midgut, only a handful develops into a mature oocyst that upon rupturing, release sporozoites into the haemocoel. Sporozoites then migrate towards salivary glands of mosquito through haemolymph. Phagocytosis and melanin deposition on the surface of invading parasites represents the major immune responses in arthropods $[23,24]$. The mechanism of these responses is not completely understood. However, various models have been proposed. Melanization of the invading ookinetes possibly results in the reduced formation of oocysts, thereby preventing transmission.

A total of 22 molecules have been identified thus far, to play a role in the melanization of ookinetes within the mosquitoes. Gene silencing studies undertaken to understand the biology of melanogenesis, revealed the role of serine proteases, such as CLIPB4, CLIPB8 and TEP1 $[10,21]$. Silencing of these serine proteases resulted in the reduction in melanization, when infected with $P$. berghei. Among the 22 genes affecting the rate of melanization, two proteins SRPN6 and TEP1 were found to affect oocyst and sporozoite counts along with melanization of ookinetes. 


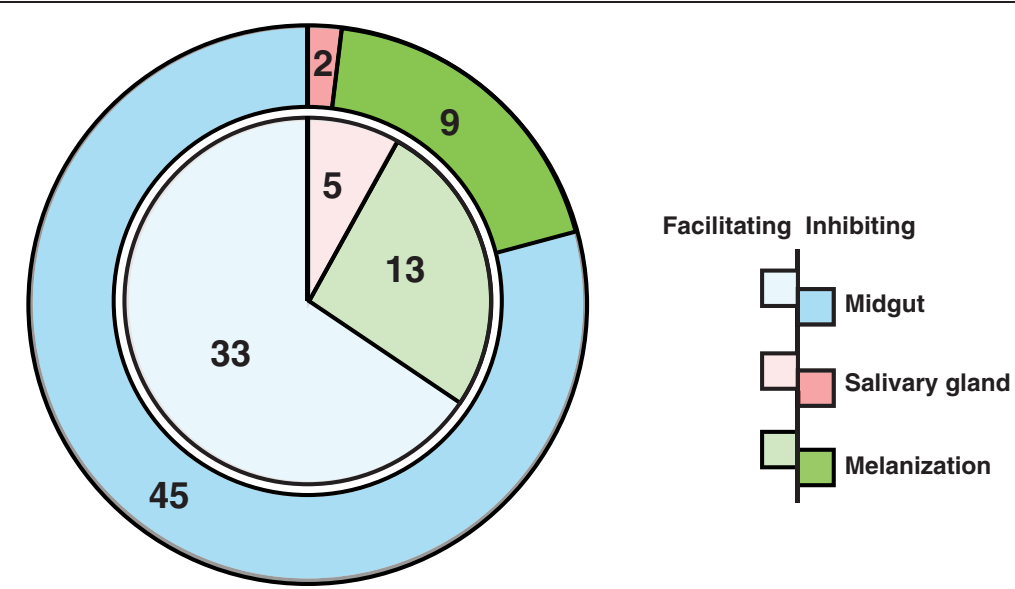

Figure 4 Pie-chart representation of molecules that promote or inhibit the Plasmodium transmission and/or development in the vector mosquito. The inner-pie in a lighter shade represents the number of molecules that are facilitating the invasion or transmission of the Plasmodium in mosquito, while the darker outer-pie represents the molecules antagonists to Plasmodium development in mosquito.

SRPN6 was reported to increase the melanization rates in refractory strains of An. gambiae, but not in susceptible G3 strain or An. stephensi. However, knockdown of SRPN6 in combination with CTL4 was reported to significantly increase the rate of melanization in susceptible G3 strain of An. gambiae [12]. Fourteen of them were found to be associated with the oocyst invasion and melanization. Silencing of seven genes including clip-domain serine proteases, such as CLIPA2 and CLIPA5 were found to increase the rate of melanization and decrease the oocyst count $[17,21,25,26]$. Knocking down of 3 genes (APL1C, LRIM1 and TEP1) showed decreased melanization of ookinetes and increased number of oocysts [8,10,16,17,20,27-34]. However, silencing of CLIPB3 and CLIPA8 resulted in the reduction of both melanization and oocyst counts (Additional files 1 and 4, Figure 3) [21]. Some investigations revealed that melanization may not necessarily affect parasite invasion, and may be involved in the disposal of dead parasites [21,35].

\section{Molecules involved in salivary gland invasion}

Sporozoites that survive mosquito immune response in the midgut lamina and haemocoel of mosquitoes migrate to salivary glands through haemolymph. Losses of up to 80 $90 \%$ sporozoites have been reported, during their migration to the salivary glands through haemolymph by various mechanisms that are not yet completely understood [36]. Effective and specific associations of sporozoite surface antigens such as TRAP, with receptors such as saglin on the salivary glands of mosquitoes initiate the salivary gland invasion by sporozoites [13]. Plasmodium responsive salivary 1 (PRS1), ESP, peptide-O-xylosyltransferase 1 (OXT1) and SRPN6 have been identified to play a crucial role in parasite invasion of both midgut and salivary glands. Silencing of PRS1, ESP, retinoid and fatty-acid binding glycoprotein
(RFABG) and OXT1 resulted in decreased oocyst and sporozoite counts (Additional files 1 and 5, Figure 3), whereas SRPN6 and TEP1 silencing resulted in an increase in production of oocysts and sporozoites [7,37-40].

The sporozoites undergo changes in their membrane proteins upon salivary gland invasion, which enable them to successfully infect the vertebral hosts [41]. Salivary gland invasion by the sporozoites and their development within is an essential step in the parasite life cycle and effective transmission of Plasmodium. Blocking of these processes could disrupt the parasite development in mosquitoes and suppress malaria transmission.

\section{Conclusions}

Blocking the vector-parasite interactions can be one of the novel approaches to control transmission of vector borne infections. Several proteins of mosquitoes have been involved in the regulation of maturation of malaria parasites within the vector. Transmission-blocking vaccine (TBV) is one of the strategies, which specifically target such proteins and block parasite invasion and survival. TBVs when injected in vertebrate host as purified antigens induce production of antibodies in the host against the corresponding antigens. These antibodies then interfere with the parasite transmission in the vector following blood meal on an infected and vaccinated individual [42]. Furthermore, engineered anti-Plasmodium immunity in transgenic mosquitoes has shown increased resistance in the vector against the pathogen [43]. Such genetically modified insects have been successful in replacing the wild type population [44].

Among the molecules listed in this study, 40 proteins were found to assist in parasite survival at different stages. Proteins which are involved in vector immunity resulted in increased number of oocysts or sporozoites upon being knocked down, showing their involvement 
in suppressing the parasite during infection. A resource of such proteins would provide a platform for other studies, to identify the potential targets and to translate the outcome as possible mechanism for malaria control. Such novel approaches along with the conventional ways of vector control and infection treatment may lead to accomplish the vision of malaria eradication.

\section{Additional files}

Additional file 1: Molecules affecting oocyst counts. The file includes the molecules that aid or inhibit the oocyst formation in mosquito midgut. The mosquito proteins that aid oocyst formation are known to be agonistic and those that inhibit the oocyst formation are known to be antagonistic in nature.

Additional file 2: Molecules that differentially affect the Plasmodium development depending on the mosquito and Plasmodium species. The file includes the list of molecules that have different effects on the malarial parasite in different mosquito and/or plasmodium species. Depending on the vector and parasite species, these molecules either aid or inhibit oocyst formation.

Additional file 3: Molecules affecting Plasmodium development studied by multiple knockdowns. The file consists of molecules, which have no or negligible effect upon silencing individually. However, when these molecules are silenced in combination with some other molecules, they affect the parasitic growth either positively or negatively

Additional file 4: Molecules affecting melanization of ookinetes. The file lists the molecules, which have been shown to be involved in the process of inhibiting or promoting the parasitic growth through ookinete melanization.

Additional file 5: Molecules affecting sporozoite counts. The file consists of molecules that promote or inhibit the development of sporozoites. The molecules that increase the sporozoite count are agonists and those that reduce sporozoite counts are known as antagonists.

\section{Abbreviations}

WHO: World Health Organization; TBV: Transmission-blocking vaccine.

\section{Competing interests}

The authors declare that they have no competing interests.

\section{Authors' contributions}

TSKP, AP, AK, HCH and NK conceived and planned the study. SKS, GD, MR, MK, MKG and AKM curated the data. SKS and GD compiled the data. SKS, $\mathrm{HCH}$ and TSKP wrote the manuscript. GD drew all diagrams. SKS prepared all tables. PS, NK, AK and AP provided critical inputs and revised the manuscript. All authors read and approved the final manuscript.

\section{Acknowledgements}

We thank the Department of Biotechnology, Government of India for research support to the Institute of Bioinformatics, Bangalore. Sreelakshmi K. Sreenivasamurthy and Gourav Dey are recipients of Junior Research Fellowship from the University Grants Commission (UGC), Government of India. Manjula Ramu and Manish Kumar are recipients of Junior Research Fellowship from the Council of Scientific and Industrial Research (CSIR), Government of India. Manoj Kumar Gupta is a recipient of Senior Research Fellowship from CSIR, Government of India. HC Harsha is a Wellcome TrustDBT India Alliance Early Career Fellow. Research in the laboratories of Akhilesh Pandey and Nirbhay Kumar is supported by NIH grants. The funding bodies had no role in the collection, analysis and interpretation of data, in the writing of the manuscript, and in the decision to submit the manuscript for publication.

\section{Author details}

'Institute of Bioinformatics, International Technology Park, Bangalore 560066, India. ${ }^{2}$ National Institute of Malaria Research, Field Station, Goa 403001, India.
${ }^{3}$ National Institute of Immunology, New Delhi 110067, India. ${ }^{4}$ Department of Tropical Medicine, Tulane University School of Public Health and Tropical Medicine, New Orleans, LA 70112, USA. ${ }^{5}$ McKusick-Nathans Institute of Genetic Medicine and Departments of Biological Chemistry, Pathology and Oncology, Johns Hopkins University School of Medicine, Baltimore, MD 21205, USA

Received: 24 January 2013 Accepted: 24 June 2013

Published: 26 June 2013

\section{References}

1. Sinka ME, Bangs MJ, Manguin S, Rubio-Palis Y, Chareonviriyaphap T, Coetzee M, Mbogo CM, Hemingway J, Patil AP, Temperley WH, Gething PW, Kabaria CW, Burkot TR, Harbach RE, Hay SI: A global map of dominant malaria vectors. Parasit Vectors 2012, 5:69.

2. Sinka ME, Bangs MJ, Manguin S, Chareonviriyaphap T, Patil AP, Temperley WH, Gething PW, Elyazar IR, Kabaria CW, Harbach RE, Hay SI: The dominant Anopheles vectors of human malaria in the Asia-Pacific region: occurrence data, distribution maps and bionomic precis. Parasit Vectors 2011, 4:89.

3. O'Brien C, Henrich PP, Passi N, Fidock DA: Recent clinical and molecular insights into emerging artemisinin resistance in Plasmodium falciparum. Curr Opin Infect Dis 2011, 24:570-577.

4. Mueller A-K, Kohlhepp F, Hammerschmidt C, Michel K: Invasion of mosquito salivary glands by malaria parasites: Prerequisites and defense strategies. Int I Parasitol 2010, 40:1229-1235.

5. Michel K, Kafatos FC: Mosquito immunity against Plasmodium. Insect Biochem Mol Biol 2005, 35:677-689.

6. Felix RC, Silveira $\mathrm{H}$ : The interplay between tubulins and P450 cytochromes during Plasmodium berghei invasion of Anopheles gambiae midgut. PLoS One 2011, 6:e24181.

7. Rodrigues J, Oliveira GA, Kotsyfakis M, Dixit R, Molina-Cruz A, Jochim R, Barillas-Mury C: An epithelial serine protease, AgESP, is required for Plasmodium invasion in the mosquito Anopheles gambiae. PLoS One 2012, 7:e35210.

8. Garver LS, Dong Y, Dimopoulos G: Caspar controls resistance to Plasmodium falciparum in diverse anopheline species. PLoS Pathog 2009, 5:e1000335.

9. Zou Z, Souza-Neto J, Xi Z, Kokoza V, Shin SW, Dimopoulos G, Raikhel A Transcriptome analysis of Aedes aegypti transgenic mosquitoes with altered immunity. PLoS Pathog 2011, 7:e1002394.

10. Molina-Cruz A, Dejong RJ, Ortega C, Haile A, Abban E, Rodrigues J, Jaramillo-Gutierrez G, Barillas-Mury C: Some strains of Plasmodium falciparum, a human malaria parasite, evade the complement-like system of Anopheles gambiae mosquitoes. Proc Natl Acad Sci USA 2012, 109:E1957-E1962.

11. Garver LS, Bahia AC, Das S, Souza-Neto JA, Shiao J, Dong Y, Dimopoulos G: Anopheles Imd pathway factors and effectors in infection intensitydependent anti-Plasmodium action. PLoS Pathog 2012, 8:1002737.

12. Abraham EG, Pinto $S B$, Ghosh A, Vanlandingham DL, Budd A, Higgs $S$, Kafatos FC, Jacobs-Lorena M, Michel K: An immune-responsive serpin SRPN6, mediates mosquito defense against malaria parasites. Proc Natl Acad Sci USA 2005, 102:16327-16332.

13. Ghosh AK, Devenport M, Jethwaney D, Kalume DE, Pandey A, Anderson VE, Sultan AA, Kumar N, Jacobs-Lorena M: Malaria parasite invasion of the mosquito salivary gland requires interaction between the Plasmodium TRAP and the Anopheles saglin proteins. PLOS Pathog 2009, 5:e1000265.

14. Schneider DS, Ghosh AK, Devenport M, Jethwaney D, Kalume DE, Pandey A, Anderson VE, Sultan AA, Kumar N, Jacobs-Lorena M: Malaria parasite invasion of the mosquito salivary gland requires interaction between the Plasmodium TRAP and the Anopheles saglin proteins. PLoS Pathog 2009, 5:e1000265.

15. Mendes AM, Awono-Ambene PH, Nsango SE, Cohuet A, Fontenille D, Kafatos FC, Christophides GK, Morlais I, Vlachou D: Infection intensitydependent responses of Anopheles gambiae to the African malaria parasite Plasmodium falciparum. Infect Immun 2011, 79:4708-4715.

16. Jaramillo-Gutierrez G, Rodrigues J, Ndikuyeze G, Povelones M, Molina-Cruz A, Barillas-Mury C: Mosquito immune responses and compatibility between Plasmodium parasites and anopheline mosquitoes. BMC Microbiol 2009, 9:154.

17. Cohuet A, Osta MA, Morlais I, Awono-Ambene PH, Michel K, Simard F, Christophides GK, Fontenille D, Kafatos FC: Anopheles and Plasmodium: 
from laboratory models to natural systems in the field. EMBO Rep 2006, 7:1285-1289.

18. Dinglasan RR, Kalume DE, Kanzok SM, Ghosh AK, Muratova O, Pandey A, Jacobs-Lorena M: Disruption of Plasmodium falciparum development by antibodies against a conserved mosquito midgut antigen. Proc Natl Acad Sci USA 2007, 104:13461-13466.

19. Blandin S, Shiao SH, Moita LF, Janse CJ, Waters AP, Kafatos FC, Levashina EA: Complement-like protein TEP1 is a determinant of vectorial capacity in the malaria vector Anopheles gambiae. Cell 2004, 116:661-670.

20. Habtewold T, Povelones M, Blagborough AM, Christophides GK: Transmission blocking immunity in the malaria non-vector mosquito Anopheles quadriannulatus species A. PLoS Pathog 2008, 4:e1000070.

21. Volz J, Muller HM, Zdanowicz A, Kafatos FC, Osta MA: A genetic module regulates the melanization response of Anopheles to Plasmodium. Cell Microbiol 2006, 8:1392-1405.

22. Vlachou D, Schlegelmilch T, Christophides GK, Kafatos FC: Functional genomic analysis of midgut epithelial responses in Anopheles during Plasmodium invasion. Curr Biol 2005, 15:1185-1195.

23. Christensen BM, Li J, Chen CC, Nappi AJ: Melanization immune responses in mosquito vectors. Trends Parasitol 2005, 21:192-199.

24. Blandin SA, Levashina EA: Phagocytosis in mosquito immune responses. Immunol Rev 2007, 219:8-16.

25. Shin SW, Zou Z, Raikhel AS: A new factor in the Aedes aegypti immune response: CLSP2 modulates melanization. EMBO Rep 2011, 12:938-943.

26. Pinto SB, Lombardo F, Koutsos AC, Waterhouse RM, McKay K, An C, Ramakrishnan C, Kafatos FC, Michel K: Discovery of Plasmodium modulators by genome-wide analysis of circulating hemocytes in Anopheles gambiae. Proc Natl Acad Sci USA 2009, 106:21270-21275.

27. Riehle MM, Xu J, Lazzaro BP, Rottschaefer SM, Coulibaly B, Sacko M, Niare O, Morlais I, Traore SF, Vernick KD: Anopheles gambiae APL1 is a family of variable LRR proteins required for Rel1-mediated protection from the malaria parasite, Plasmodium berghei. PLOS ONE 2008, 3:e3672.

28. Mitri C, Jacques JC, Thiery I, Riehle MM, Xu J, Bischoff E, Morlais I, Nsango $\mathrm{SE}$, Vernick KD, Bourgouin C: Fine pathogen discrimination within the APL1 gene family protects Anopheles gambiae against human and rodent malaria species. PLOS Pathog 2009, 5:e1000576.

29. Riehle MM, Markianos K, Niaré O, Xu J, Li J, Touré AM, Podiougou B, Oduol F, Diawara S, Diallo M, Coulibaly B, Ouatara A, Kruglyak L, Traoré SF, Vernick KD: Natural malaria infection in Anopheles gambiae is regulated by a single genomic control region. Science 2006, 312:577-579.

30. Povelones M, Waterhouse RM, Kafatos FC, Christophides GK: Leucine-rich repeat protein complex activates mosquito complement in defense against Plasmodium parasites. Science 2009, 324:258-261.

31. Osta MA, Christophides GK, Kafatos FC: Effects of mosquito genes on Plasmodium development. Science 2004, 303:2030-2032.

32. Dong Y, Aguilar R, Xi Z, Warr E, Mongin E, Dimopoulos G: Anopheles gambiae immune responses to human and rodent Plasmodium parasite species. PLoS Pathog 2006, 2:e52.

33. Dong Y, Dimopoulos G: Anopheles fibrinogen-related proteins provide expanded pattern recognition capacity against bacteria and malaria parasites. J Biol Chem 2009, 284:9835-9844.

34. Rono MK, Whitten MM, Oulad-Abdelghani M, Levashina EA, Marois E: The major yolk protein vitellogenin interferes with the anti-Plasmodium response in the malaria mosquito Anopheles gambiae. PLoS Biol 2010, 8:e1000434.

35. Schnitger AK, Kafatos FC, Osta MA: The melanization reaction is not required for survival of Anopheles gambiae mosquitoes after bacterial infections. J Biol Chem 2007, 282:21884-21888.

36. Hillyer JF, Barreau C, Vernick KD: Efficiency of salivary gland invasion by malaria sporozoites is controlled by rapid sporozoite destruction in the mosquito haemocoel. Int J Parasitol 2007, 37:673-681.

37. Chertemps T, Mitri C, Perrot S, Sautereau J, Jacques JC, Thiery I, Bourgouin C, Rosinski-Chupin I: Anopheles gambiae PRS1 modulates Plasmodium development at both midgut and salivary gland steps. PLoS One 2010 5:e11538.

38. Armistead JS, Wilson IB, van Kuppevelt TH, Dinglasan RR: A role for heparan sulfate proteoglycans in Plasmodium falciparum sporozoite invasion of anopheline mosquito salivary glands. Biochem J 2011, 438:475-483.

39. Pinto SB, Kafatos FC, Michel K: The parasite invasion marker SRPN6 reduces sporozoite numbers in salivary glands of Anopheles gambiae. Cell Microbiol 2008, 10:891-898.
40. Ramakrishnan C, Rademacher A, Soichot J, Costa G, Waters AP, Janse CJ, Ramesar J, Franke-Fayard BM, Levashina EA: Salivary gland-specific $P$. berghei reporter lines enable rapid evaluation of tissue-specific sporozoite loads in mosquitoes. PLOS ONE 2012, 7:e36376.

41. Kappe SH, Kaiser K, Matuschewski K: The Plasmodium sporozoite journey: a rite of passage. Trends Parasitol 2003, 19:135-143.

42. The malERA Consultative Group on Vaccines: A research agenda for malaria eradication: vaccines. PLoS Med 2011, 8:e1000398.

43. Meredith JM, Basu S, Nimmo DD, Larget-Thiery I, Warr EL, Underhill A, McArthur CC, Carter V, Hurd H, Bourgouin C, Eggleston P: Site-specific integration and expression of an anti-malarial gene in transgenic Anopheles gambiae significantly reduces Plasmodium infections. PLOS One 2011, 6:e14587.

44. Alphey L, Nimmo D, O'Connell S, Alphey N: Insect population suppression using engineered insects. Adv Exp Med Biol 2008, 627:93-103.

doi:10.1186/1475-2875-12-216

Cite this article as: Sreenivasamurthy et al: A compendium of molecules involved in vector-pathogen interactions pertaining to malaria. Malaria Journal 2013 12:216

\section{Submit your next manuscript to BioMed Central and take full advantage of:}

- Convenient online submission

- Thorough peer review

- No space constraints or color figure charges

- Immediate publication on acceptance

- Inclusion in PubMed, CAS, Scopus and Google Scholar

- Research which is freely available for redistribution 